\title{
Conceptual Modeling of Multimedia Search Applications Using Rich Process Models
}

\author{
Alessandro Bozzon, Marco Brambilla, and Piero Fraternali \\ Politecnico di Milano, \\ Piazza Leonardo Da Vinci, 32 - 20133 Milano, Italy \\ \{alessandro.bozzon, marco.brambilla, piero.fraternali\}@polimi.it
}

\begin{abstract}
With the advent of the Web, search has become the prominent paradigm for information seeking, both across the online space and within enterprises. Search frameworks and components can be used to build search-based applications in the most diverse vertical fields. This paper explores Model Driven Development and model transformations as a paradigm for developing search-based applications, considered as process- and content-intensive applications. A rich process model, obtained by extending BPMN, is proposed as the starting point of development, followed by a set of semi-automatic model transformations that lead from the conceptualization of requirements to the running code.
\end{abstract}

\section{Introduction}

With the diffusion of the Web, search has become the predominant paradigm for addressing the information needs of users. Nowadays search is not only confined to the online information space, but has assumed a fundamental role also in enterprise application integration, as a practical means to grant unified access to the vast collections of structured, semi-structured, and unstructured contents that constitute the core of modern businesses. This brings about the notion of Search-Based Application (SBA), i.e., an application in which searching constitutes the predominant user interface paradigm.

Unlike monolithic search engines, SBAs are mostly tailor-made evolving solutions, because the nature of the searched information and of the user needs vary considerably across different business sectors [13] and in time. The main factors of SBA complexity and variability stem from: content, which can span distributed database, document, and audiovisual materials; content processing, which involves crawling, transcoding, analysis, annotation, and indexing 1 ; and querying, which must support multiple paradigms like keyword search, contentbased similarity search (query by image, by sketching, by humming, etc), query refinement and enrichment based on the profile of the user and of her community.

The thesis of this paper is that SBA development demands for the same evolution in methods and tools that has characterized in the recent past the progress

\footnotetext{
${ }^{1}$ Annotations are the metadata extracted by analyzing the content: e.g., frequent terms for documents, or features for images, audio, and video.
} 
of Web Engineering [8] for "classical" Web applications. We claim that SBAs demand for novel approaches and tools, which combine the benefits of processdriven and data-driven design methodologies, while adding new solutions to specific needs of SBAs. SBAs are undergoing a growth towards complex, tailormade, personalized, multichannel, and adaptive applications, while retaining a specific flavor, due to the prevailing role of search: they must integrate a complex front-end (for query submission and result presentation) with a complex back-end (content provisioning, annotation, indexing, and distributed query execution). Both aspects involve a lot of search-specific aspects, that cannot be treated by usual approaches: the query and the result list are subject to a specific life-cycle: they must be obtained, reformulated, integrated or enriched, and stored. The same is true for content, which must be provisioned, transcoded, analyzed, annotated, and indexed. These processes vary based on requirements, which may evolve over time or change depending on the business sector.

The approach proposed in this paper is based on the paradigm of Model Driven Development (MDD), where models are the core artifacts of the application life-cycle and model transformations progressively refine models to achieve an executable version of the system. The core contribution of the paper is twofold: 1) the set of models that can represent the core elements of a SBA; 2) a set of model transformations from the initial model to the executable system.

To cope with the process-intensive nature of the main SBAs' interactions (i.e., content analysis, query management, etc.), we advocate the use of a Rich Process Model [20], obtained by extending BPMN with search-specific primitives, as the starting point of both the front-end and the back-end SBA specification. In the terminology of MDA, the Rich Process Model can be seen as a Computation Independent Model (CIM), which specifies SBA requirements. Such Rich Process Model can be semi-automatically transformed into an Application Model, encoded using WebML [3], a Platform Independent Model (PIM) that exploits SOA and Web interfaces as a technical space to design data-intensive Web applications. Finally, Java 2 application code can be generated as a Platform Specific Model (PSM), through an extended version of an existing tool [19]2.

To our knowledge, the proposed approach is the first that addresses the Model Driven Development of SBAs through semi-automatic model transformations. General-purpose Web Engineering methodologies have been applied to dataintensive [12]316 and process-intensive applications [1|15|18|10]. However, none of these approaches has focused on the specific requirements of SBAs nor implemented the model transformations needed for turning a rich process model into an application.

The paper is organized as follows: Section 2 summarizes the main characteristics of SBAs; Section 3 overviews the SBA models and transformations; Sections 4 and 5 respectively detail the domain model and the process model; Section 6 discusses the CIM to PIM model transformation; Section 7 presents the

${ }^{2}$ A detailed discussion about the chosen modeling and deployment technologies is out of the scope of this paper. The reader may refer to [320] for further details. 
application model; Section 8 reports on implementation experience; Section 9 reviews the related works; and finally Section 10 concludes.

\section{Search-Based Applications}

Figure 1 shows the multi-tier reference architecture of a SBA. The Presentation Tier deals with the rendition of the user interface whereby users can formulate their information need as a query to the system. This level must allow multiple ways of expressing queries: e.g., by means of keywords or by the submission of examples of content similar to the desired ones. The Frontend Business Logic Tier is responsible of: 1) receiving the query from the Presentation Tier, preprocessing it, and submitting it for execution to the back-end tier; 2) receiving the result list, postprocessing it and forwarding it to the Presentation Tier for rendition. Examples of query preprocessing are: linguistic or semantic treatment of keywords (e.g., stemming, language identification), query personalization with user's preferences. Examples of result post-processing include: filtering based on access rights, enrichment with external non-indexed information (e.g., content previews), etc. The Backend Business Logic Tier manages content acquisition, feature extraction, encoding of features as content annotations, and indexing of such annotations in one or more search engines. It also orchestrates the execution of the query, by brokering it across one or more search engines.

As shown in Figure 1, the SBA architecture supports two major flows of activities, which are the main subject of SBA design: 1) The Query and Result Presentation (QUIRP) process, which encompasses query preprocessing, query execution, and results post-processing; 2) The Content Provision and Annotation (CPA) process, which gets content from its original location and makes it available to the search engines, together with the appropriate annotations. The rest of the paper will show how to formalize such processes and their supporting data, together with the model transformation to progressively obtain an executable application embodying the desired QUIRP and CPA flows.

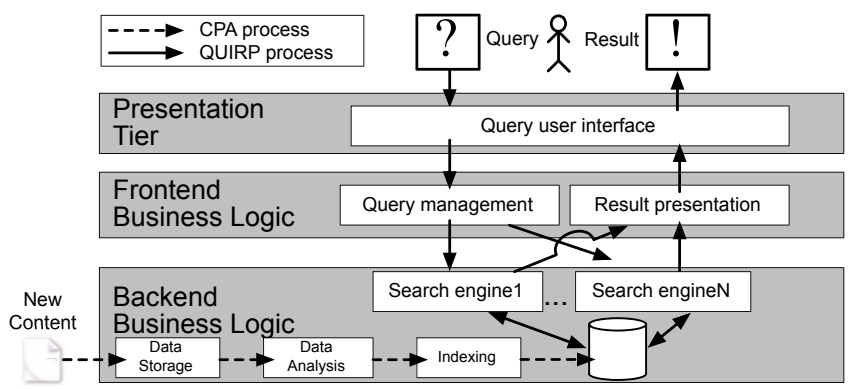

Fig. 1. Architecture and Processes of a SBA 


\section{Models and Transformations}

Figure 2 outlines the models and transformations involved in SBA design. Initially, requirements are conceptualized in 1) a Domain Model, which formalizes, using any object-oriented notation (e.g., UML), the essential objects managed by the SBA and their relationships, and 2) a Process Model describing the QUIRP and CPA workflows in terms of activities, precedences, and data flows. We describe process models by means of BPMN diagrams 20, coherently with the BPDM metamodel 3 , which has been extended with activity and parameter typing. Domain objects support the QUIRP process, the CPA process, and process enactment. The link between the domain and process models is established by the type of objects that flow between activities, and by classifying each activity based on its behaviour.

The Domain Model and Process Model are subject to a first (CIM to PIM) transformation, which produces the Application Model and Process Metadata. The Application Model expresses the design of the SBA application as a set of coordinated services that implement the QUIRP and CPA processes, possibly augmented with hypertext interface to support human-directed activities. We encode the application model in WebML [3], which allows the seamless specification of hypertexts and services. The Process Metadata, instead, represents a logical view of the constraints between activities, useful for encapsulating the process control logic in the Application Model. Explicit process metadata simplify the Application Model, because process advancement control logic can be encapsulated in one dedicated operation, which exploits process metadata. The generated Application Model can be refined manually by the designer, to add domain-dependent information on the execution of activities.

The manually refined Application Model is the input of a second (PIM to PSM) transformation, which produces the code of the application for a specific technological platform. The Implementation Model (PSM) includes all the needed software artifacts (i.e., running code, database schema and instances, XML configuration files, and so on). The PSM we produce is in the Java2 technical space, based on the MVC framework and relational technology. The PIM to PSM transformation is completely automated thanks to an extension added to the commercial tool WebRatio [19].

These transformations extend the model-driven design of Web applications from business process specification proposed in [1].

\begin{tabular}{|c|c|c|c|c|}
\hline $\begin{array}{r}\text { Domain Model } \\
\text { (CIM) }\end{array}$ & $\begin{array}{c}\text { ATL } \\
\text { Transf. }\end{array}$ & $\begin{array}{r}\text { Process Metadata } \\
\text { (PIM) }\end{array}$ & $\begin{array}{l}\text { Java } \\
\text { Transf. }\end{array}$ & $\begin{array}{r}\text { Ru } \\
\text { Search- }\end{array}$ \\
\hline $\begin{array}{r}\text { ocess Model } \\
(\mathrm{CIM})\end{array}$ & (CIM t & (P & (PIM & (F \\
\hline
\end{tabular}

Fig. 2. Model to model transformations from CIM to PIM to PSM

${ }^{3}$ http://www.omg.org/spec/BPDM/1.0/ 


\section{Domain Model}

The domain model formalizes data and metadata supporting the QUIRP and CPA processes, and comprises the sub-models shown in Figure 3 . For the sake of illustration, from now on we assume a SBA case study dealing with multi-modal audiovisual search, supported through three paradigms: matching keyword over content annotation, similarity to an audio sample, and similarity to a person's face.

The main SBA domain sub-models (Figure 3) are: 1) the Content Model, which defines the Content Items of interest for the search, associated to Annotations and other collateral resources (e.g., transcoded version of audio/video); 2) the Query Model, which specifies the admitted types of Query (e.g., face similarity, audio similarity); 3) the Result Model, which defines the structure of the ResultSet; 4) the Index Model, which represents the information that is stored in the Index of the managed search engines; 5) the Tracking Model, which describes the ActivityInstances executed during the enactment of a specific case of the QUIRP and CPA processes (processCase); 6) the User Model, which reflects the User profile and roles (Groups).
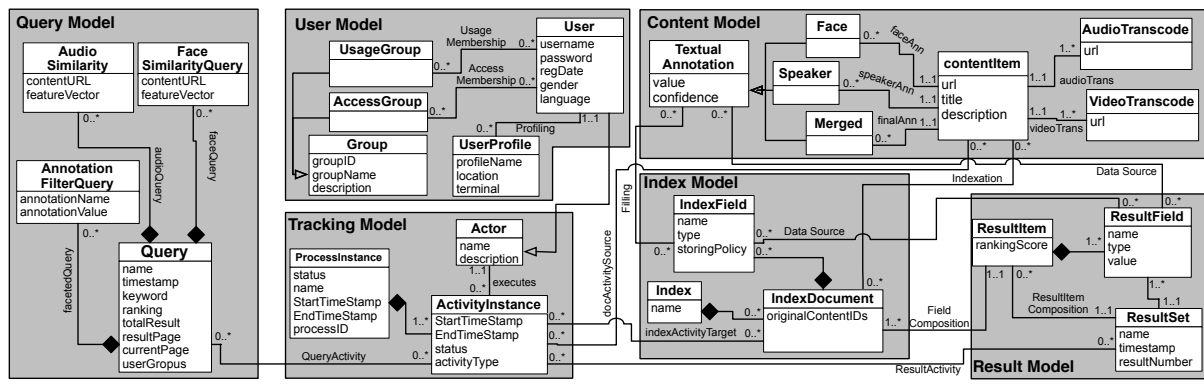

Fig. 3. Example of Domain model for an audiovisual SBA

\section{Process Model}

The Process Model exploits the BPMN notation 20, extended with SBA-specific information to enable a more precise model transformation towards PIM and PSM models. The classification of activities based on their semantics and the precise typing of data flows permit the generation of PIMs that are very close to a complete solution usable for generating the code of the application.

Figure 4 shows the graphical notation of the extended BPMN activity. An activity is associated with a Type (1), which denotes the kind of processing performed (e.g., Data Analysis), and possibly a set of refined SubTypes (2) (e.g., Music Genre Analysis). The activity performs the processing implied by all the associated subtypes, combined in a domain-dependent way. An activity has a (possibly empty) set of input parameters (3) and output parameters (4). The actual values of input parameters can be assigned from one or more input objects, 


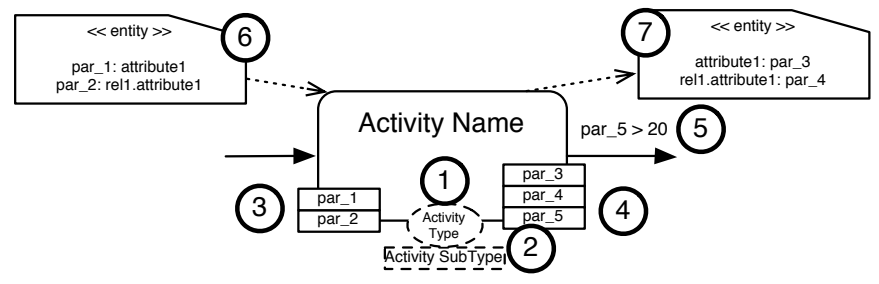

Fig. 4. Extended activity notation

associated to incoming links; the output parameters can take values from some objects produced or modified by the activity. The output flow of an activity can be associated to a guard condition (5), which is an OCL Boolean expression over the values of the output parameters. The target activity of the guarded link can be executed only if the activity source of the link has completed and the condition evaluates to true. Input and output objects (6) (7) correspond to instances of the classes specified in the Domain Model.

Activities in the CPA process can have one of the following types: Retrieval, Transformation, Analysis, Aggregation, Indexation, and Storage. For the sake of conciseness, from now on we will only discuss the QUIRP process 4 .

Activities in the QUIRP process can have one of the following types:

- Query specification (QS): denotes the submission of a query;

- Query management (QM): denotes the manipulation of the query, e.g., for separating parts to be assigned to different search engines;

- Search (S): denotes the actual execution of the query by a search engine;

- Result Aggregation (RA): the activity merges two or more result sets provided by different search engines;

- Result Enrichment (RSE): the activity personalizes the results based on social knowledge and user preferences, inferred from previous searches by the same users or other users connected to her;

- Result Presentation (RP): the activity formats results according to the need of the presentation tier.

Such a categorization do not claim for completeness, but it stems from the authors' experience in the field. Nonetheless, new activity types can be added at will, without affecting the validity nor the generality of our approach.

Figure 5 shows an example of QUIRP process where the query can be specified according to three different modalities: by keyword, by audio recognition of the speaker, and by face recognition of the appearing characters. These options are specified as SubTypes to be performed within the Query Specification activity. The Query Management activity reshapes the query and assigns each part to the respective Search Activity. Results are then aggregated, enriched and personalized, and finally presented to the user, that can possibly decide to

${ }^{4}$ An extended version comprising also discussion and examples of CPA process can be found at http://home.dei.polimi.it/bozzon/ICWE2009/SBAMDD_ICWE2009.pdf 


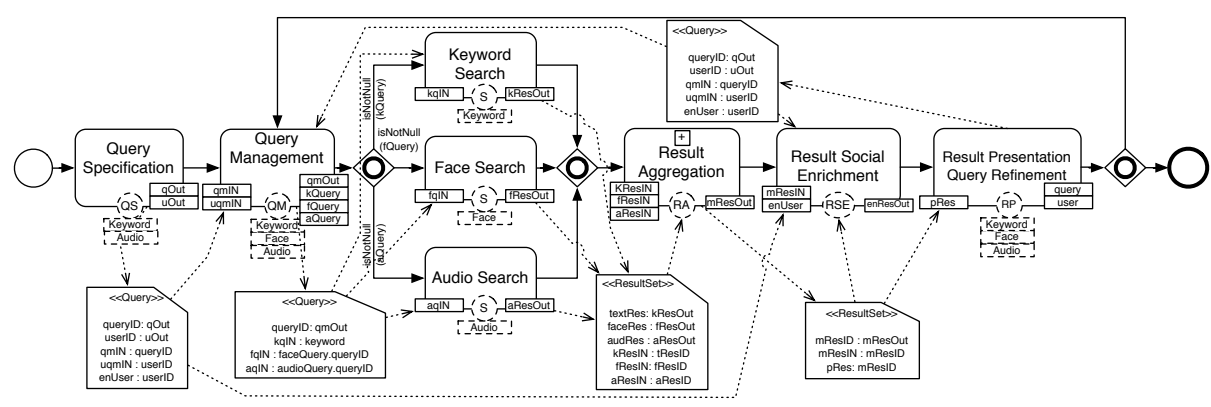

Fig. 5. Process models example: the QUIRP process

refine the query, thus triggering again the Query Management, for addressing the refinement parts of the query.

\section{CIM-PIM Model Transformations}

The transformation from CIMs to PIMs consists of two sub-transformations:

- BPM to Process Metadata: the business process specifications are transformed to instances of a relational representation of the Process Metamodel shown in Figure 6, for enabling runtime control of activity precedence constraints;

- BPM to Application Model: the business process is transformed to a coarse Application Model, which can be subsequently refined by the designer.

\subsection{Process Metadata generation from BPMN}

The transformation from BPMN to the relational representation of Process Metadata adheres to the following guidelines:

- each BPMN process is transformed to a Process instance;

- each BPMN activity is transformed to a Activity Type instance;

- each BPMN flow arrow is transformed to a nextActivity/previousActivity relation;

- each guard condition is transformed to a Condition instance, whose OCL expression is the expression of the original guard condition;

- each gateway is transformed to a Condition instance (in conjunction with the possibly existing guard conditions on the incoming and outgoing arrows).

The conditions generated for the gateway elements are defined according to the BPMN semantics:

- AND-splits allow a single thread to split into two or more parallel threads, which proceed autonomously. The condition over the workflow structure is the TRUE expression, because as soon as the preceding activity is finished, all the branches can start independently. 


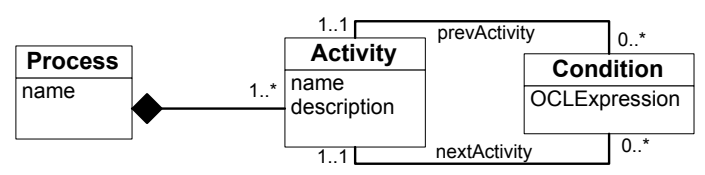

Fig. 6. Metadata describing activity precedence constraints

- XOR-splits represent a decision point among several mutually exclusive branches. Its condition will define that one of the alternatives can start iff all the others haven't started yet.

- OR-splits represent a decision for executing one or more branches. The condition is the TRUE expression, because one or more branches can start independently.

- AND-joins specify that an activity can start iff all the incoming branches are completed. The associated condition will allow execution of the next activity iff all the previous ones are completed.

- XOR-joins specify that the execution of a following activity can start as soon as one activity among the incoming branches has been terminated. The condition will check this situation to allow the execution of the next activity.

- OR-joins specify that the execution of the following activity can start as soon as all the started incoming branches have been terminated. The condition will check this situation.

Process Metadata generation has been formalized as an ATL transformation from the BPDM metamodel to the Process Model of Figure 6

\subsection{WebML Model Generation from BPMN}

The transformation from Process Models to WebML coarse models of services and hypertext interfaces considers modeling dimensions like actor type (human or automatic), process distribution, managed data objects, and exception handling. The application models produced by the transformation still need manual refinement, to add domain-specific elements that cannot be expressed even in the enriched BPMN notation. However, by exploiting information about the activity type/subtype, a first-cut application model can be generated.

WebML Workflow Primitives. A WebML application model can be either an hypertext model or a service model. An hypertext model, called site view, is a set of navigable pages comprising static or dynamic content elements. Pages, content elements, and executable operations are connected in a graph structure by links, which allow navigation and operation execution. A service model, called service view, denotes the orchestration of multiple service executions; it is expressed as a graph of operations denoting the receipt of a message, the sending of a message, and the execution of a piece of business logic.

${ }^{5}$ A sample ATL fragment that calculates the condition of a XOR split can be found at home.dei.polimi.it/bozzon/ICWE2009/SBAMDD_ICWE2009.pdf 
Both site views and service views can be subjected to the constraints of a process model, by exploiting ad hoc operations that denote the starting and closing of an activity (Start and End units), the storage and retrieval of parameter values (Assign and Retrieve units), and the computation of the next enabled activities given the current state of the workflow (Next unit). These units are discussed in the paper [1, except for the Next unit, which has been defined in this work.

A specific component (Next unit) encapsulates the process control logic: it exploits the information stored in the Process Metadata and in the Tracking Domain Model to calculate the current process status and the enabled transitions. Given the activityID of the ActivityInstance just terminated, the current caseID (process instance), and the conditionParameters (the values required by the conditions to be evaluated), the Next unit queries the Process Metadata to find all the process constraints and enables the execution of the proper activities. If the activities are automatic, they are immediately started. If they involve human interaction, the corresponding hypertext are enabled.

Process Transformation. The Process transformation from BPMN to WebML consists of three main rules: the Process transformation rule, addressing the whole process; the Activity transformation rule, managing starting, closing, and parameters of each activity; and the Business logics transformation rule, addressing the internal logic of the activities, based on the activity type/subtype.

The outcome of the Process transformation rule is a WebML model that comprises: 1) the process initiation and termination logic, generated from the Start Process and End Process BPMN events; 2) a site view or service view for each BPMN pool; 3) a set of hypertext pages or a graph of services for each BPMN activity.

The WebML model for process initiation (process termination) is defined based on the type of BPMN start (end) event; it creates (consumes) data objects, supplied by the process initiator in order to run the case (returned as a result of process termination). If the start (end) event is a BPMN message events, we assume the process to be exposed as a Web service, with an invocation (response) interface comprising a parameter for every consumed (produced) data object. Otherwise, the transformation produces an hypertext page for inputting the objects needed for initiating the process (for accessing the objects resulting from process termination).

The Activity transformation rule is based on the BPMN activity specification, taking into account aspects like the actor enacting the activity (e.g., a user or the system), pre- and post-condition specification, as well as exception management. For each BPMN activity, a set of WebML primitives is generated, as shown in Figure 7, The WebML model is embodied within a siteview named as the BPMN pool containing the activity, and is composed of seven blocks:

- Inception, evaluating the set of pre-conditions for the activity through a Switch unit. If the condition holds, then the execution can proceed.

- Initiation, devoted to starting the activity. It comprises a Start Activity unit and, for each consumed data object, a Retrieve unit properly configured for 


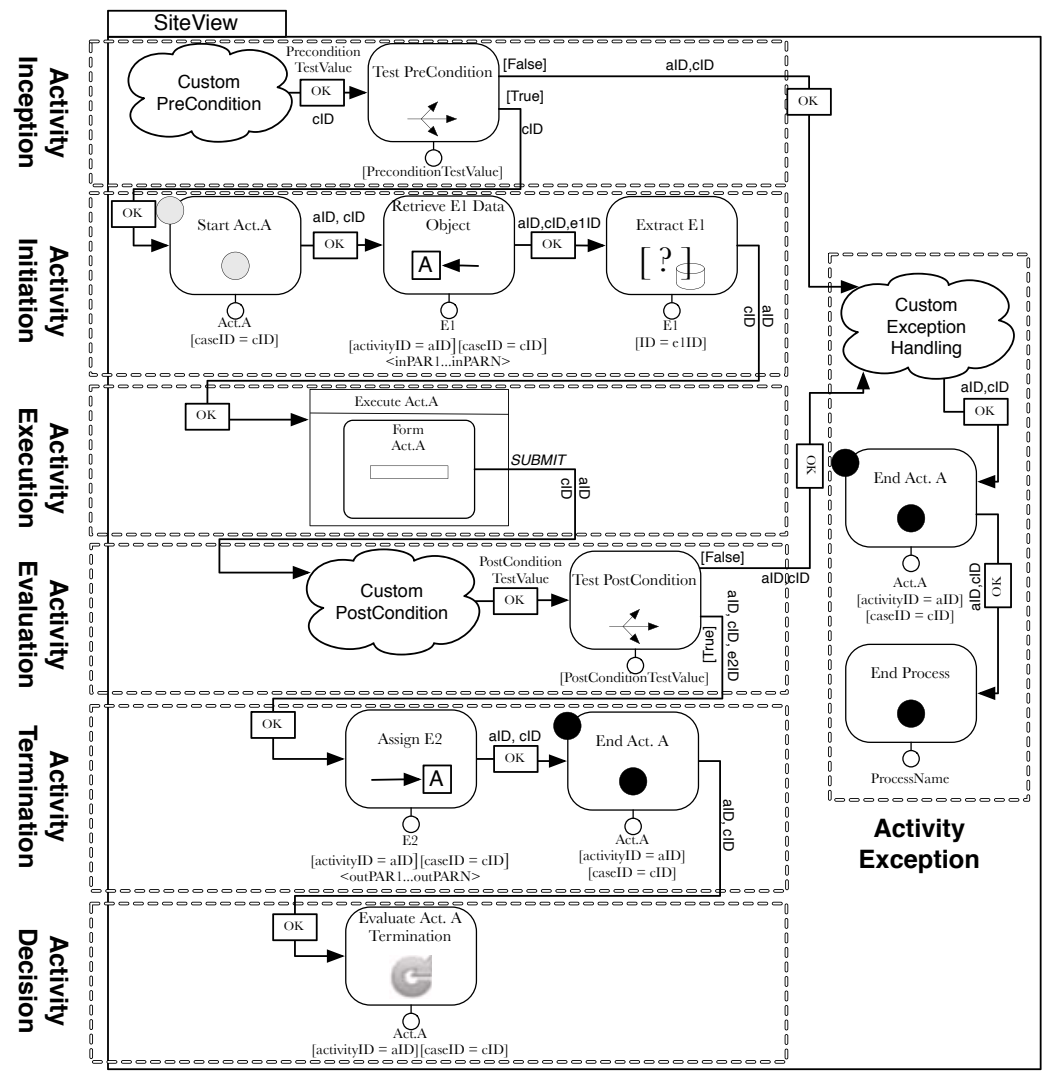

Fig. 7. Skeleton of the result of the BPMN-WebML activity transformation

extracting the needed parameters and a Selector unit retrieving the work items;

- Execution, depending on the business logic enforced by the specific activity. If no Activity type and subtype are specified at the BPMN level, it is possible to generate only a functionally void set of WebML units, which the designer will later substitute with the needed ones. Otherwise, the Business logics transformation rule is in charge of producing a more refined model;

- Evaluation, evaluating the set of post-conditions for the activity, similarly to pre-conditions.

- Termination, closing the activity. For each created data object, an Assign unit is defined, followed by an End Activity unit.

- Decision, defining the advancement of the process, through the Next unit.

- Exception, handling exceptional events, by means of compensation activities or by interrupting the process. In our transformation, exceptions are checked during pre- and post-condition evaluation. 
The Business logic transformation rule is in charge of generating the execution part of the activity. The generated WebML fragment depends on the type of actor, on the distribution of actors, and on the type and operations:

- For user-enacted activities, activity execution consists of a user-browsable hypertext, while for automatic activities it consists of a service view.

- Process activities assigned to distributed actors require a communication modeled in BPMN by means of message events. Our transformation assumes Inter-actor communication to be based on Web Services.

- For each Activty Type, a specific WebML hypertext pattern is defined as general purpose implementation. Moreover, if one or more Subtypes are specified, the pattern is composed by one or more subpatterns, each describing the corresponding operation. For instance, an Activity of type QuerySpecification will be transformed to a submission page for the search criteria. If the type is detailed by the AudioSearch and TextSearch operations, the input interface in the hypertext will be generated accordingly.

The whole approach is specified by an ATL transformation organized into the three above specified rules: a Process transformation rule generates the process level actions and then invokes the generic Activity rule that manages untyped activities (by generating an activity skeleton as shown in Figure 7). A set of type-specific Activity rules inherit from the general transformation and refine it according to the Activity Type. These rules also consider the SubType and generate the appropriate hypertext elements, according to a composition logics specific for each Activity Type. A skeleton of the generic Activity transformation is available in the extended version of this paper 6 .

\section{Application Model}

The result of the CIM to PIM transformation consists of a coarse Web application model, which needs to be manually refined by the designer. The generated model complies with the already existing WebML metamodel[2, extended with the new SBA primitives. This section exemplifies the refined WebML application models of two representative QUIRP process activities derived from the BPMN example of Figure 5 .

Figure [8(a) depicts the WebML model for the Query Specification activity, which involves the user interaction and therefore is generated as a WebML hypertext. The Query Specification unit (1) sets the activity status to Active. The Search Page contains a form for each query modality defined as Activity SubType in the process model. In our example, the Keyword (3) and Audio Content file upload (2) forms are created starting from the activity subtypes "Keyword" and "Audio". By navigating the Submit link (L1), the user submits his search criteria and triggers the the definition of the new Query instance (4) and of the associated Audio Similarity term $(5,6)$. The Assign Query unit (7) assigns

${ }^{6}$ home.dei.polimi.it/bozzon/ICWE2009/SBAMDD_ICWE2009.pdf 
(a) - Query Specification Activity

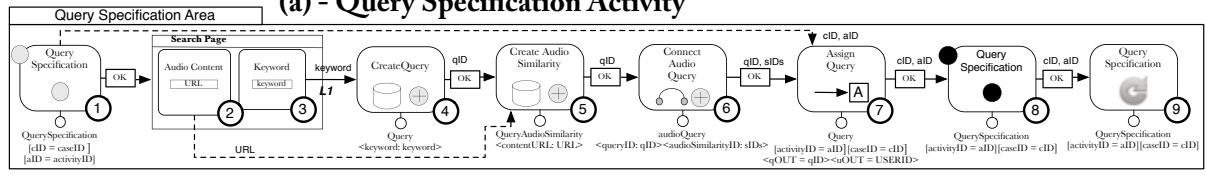

(b) - Query Management Activity

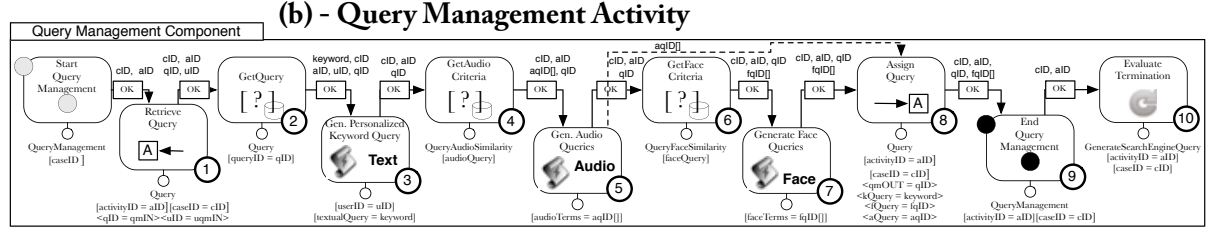

Fig. 8. WebML models of (a) Query specification and (b)Query management activities

the created query and parameters to the next activities. Finally, the activity ends $(8,9)$.

Figure 8(b) depicts the Query Management activity which creates the actual query terms to be submitted to the different search engines. After retrieving Query and User (1), the Get Query (2) and Gen. Personalized Keyword Query (3) units address the query keyword terms, by respectively extracting them from the current Query instance (2) and generating the textual search engine's query (3), where also user personalization is applied. Then, the QueryAudioSimilarity $(4,5)$ and QueryFaceSimilarity $(6,7)$ query terms are processed, where the lowlevel features are calculated and translated in the respective search engines query languages. Finally, the Assign Query unit (8) sets the values to be provided as output parameters, the current activity is closed (9), and the Next unit (10) triggers the next activities. For the QUIRP process of Figure 5, the Next unit must evaluate an OR gateway, which means that the activities (i.e., Keyword Search, Face Search and Audio Search) for which a query term is defined are triggered.

\section{Implementation and Experience}

This section reports our experiences in the extension of WebRatio 57, a CASE tool supporting WebML design and code generation of industrial Web applications. The extension regarded all three major component of the WebRatio suite: the modeling editor GUI, the code generator, and the runtime environment.

The modeling GUI has been extended by: 1) creating an online workflow editor implementing the SBA-specific extensions of BPMN 8; 2) adding the SBAspecific units to the WebRatio design environment. The code generator has been extended in two directions: 1) the CIM to PIM transformation has been implemented within the toolsuite, to allow seamless transformation of BPMN models to WebML and to the Process Metadata. 2) the PIM to PSM transformation has been enriched by the Process Metadata transformation towards platform

\footnotetext{
${ }^{7}$ http://www.webratio.com.

${ }^{8}$ http://home.dei.polimi.it/mbrambil/SBAwfEditor.htm
} 
specific database tuples and by extending the existing j2EE code generator to cover the new WebML primitives. The runtime counterpart of the new primitives has been created too. Since at runtime search applications have to be very efficient, attention must be paid to the implementation issues. SBA applications must exploit ad-hoc optimizations which reduce (or ignore) separation of concerns between architectural modules or execution steps. This issue is partially addressed by our work through the activity subtype concept: if two or more subtypes are declared for one activity, their combination is built by considering as many optimizations as possible. On the opposite, at the moment no generation of optimized combination of separate activities is implemented.

To validate the approach, we have applied it in the context of PHAROS 4 (Platform for searcHing of Audiovisual Resources across Online Spaces), an EU Integrated Project, whose peculiarity consists in being an open framework for developing audiovisual search solutions. Every functionality of the architecture is conceived to be pluggable, according to the SOA paradigm. Our contribution has been twofold: we fostered the adoption of a MDD approach for the specification of the PHAROS platform and we applied our design method for the development of both QUIRP and CPA prototype components that have been plugged into the Pharos platform. Tangible results can be found in the project's Website 9 .

\section{Related Work}

Even if several methodologies and tools exist for Model Driven Development of general-purpose and vertical applications, very few works explored the construction of search-based applications using models and model transformations. Some proposals 21 [5] offer frameworks based on design patterns for meta-search engines or vertical search engines. The idea is to associate the main components of a search engine (e.g., crawlers, text-filters) with reference UML design patterns, to be used in application design. However, the dynamic behavior of SBAs is not captured and no model transformation or code generation capability is provided. Ferreira at al. 6] introduce IRML, Information Retrieval Modeling Language, a formalism based on the UML extension mechanisms, that include a library of modules and code generation based on XML transformations. However, there is no formalization of the composition of IRML modules.

With respect to the above-mentioned works, the approach in this paper goes a step forward: it not only proposes a (data- and process-centric) model of the application requirements, but also formalizes a model-to-model transformation yielding a PIM amenable to be refined by the application designer and to be automatically transformed into running code.

SBA development could be considered as a special case of process- and datacentric application design. Several MDD methods have explored the integration of business process and Web application modeling. The Process Modeling language (PML) 14, for instance, is an early proposal for the automatic generation of simple Web-based applications exploiting process specifications. Koch et al.

${ }^{9}$ http://www $\cdot$ pharos-audiovisual-search.eu/ 
[10] approaches the integration of process and navigation modeling in the context of UWE and OO-H. The convergence between the two models focuses on the requirement analysis phase, where standard UML constructs are used. The design of the application model, instead, is separated. In our work, both approaches are considered: like in UWE, we preserve the process model as an additional domain model; as in $\mathrm{OO}-\mathrm{H}$, we provide semi-automatic generation of navigational skeletons directly from the process model.

The approach proposed by Torres and Pelechano [17] leverages BPM and OOWS 7] to model process-centric applications; model-to-model transformations are used to generate the Navigational Model from the BPM definition and model-to-text transformations can produce a WS-BPEL process definition. In contrast, our work enriches the representation of the business process with datacentric features and with typed activities, so to encode in the BPMN model more knowledge exploitable in the automatic generation of application models.

Liew at al. 11 presents a set of transformations for automatically generating a set of UML artifacts from BPM. Jonkers et al. [9] presents a more pragmatic implementation of Model-Driven Architecture (MDA) in order to provide semiautomatic generation of enterprise applications starting from business process models, by extending some existing BPM and MDA tools.

With respect to the literature on business process integration within generalpurpose Web Engineering methods, our work differs in its specific focus on SBAs, highlighting the core processes behind these solutions and demonstrating applicability of how data-centric and process-centric MDD methods.

\section{Conclusions}

In this paper we have shown how to exploit MDD methods and tools to support the development of search based Web applications. We proposed a top-down design approach that combines the benefits of (extended) business process design with the advantages of a domain specific language for the Web. We formalized a set of domain-specific BPMN extensions and a set of MDA transformations, thus covering the requirements of SBAs. Future research includes the definition of: a more complete CIM to PIM transformation; the transformation rules of a wider set of Activity Types/Subtypes; and algebraic representation of SBA processes, in order to define a verification framework of search-specific properties.

Acknowledgments. This work is partially supported by the Pharos project funded by the EU within the VII FP. We wish to thank all the partners of the project for the fruitful collaboration.

\section{References}

1. Brambilla, M., Ceri, S., Fraternali, P., Manolescu, I.: Process modeling in web applications. ACM TOSEM 15(4), 360-409 (October 2006)

2. Brambilla, M., Fraternali, P., Tisi, M.: A metamodel transformation framework for the migration of WebML models to MDA. In: CEUR-WS.org, (ed.) MDWE 2008, CEUR Proceedings, vol. 389, pp. 91-105 (2008) 
3. Ceri, S., Fraternali, P., Bongio, A., Brambilla, M., Comai, S., Matera, M.: Designing Data-Intensive Web Applications. Morgan Kaufmann Publishers Inc, San Francisco (2002)

4. Debald, S., Nejdl, W., Nucci, F., Paiu, R., Plu, M.: Pharos platform for search of audiovisual resources across online spaces. In: CEUR-WS.org. (ed.) SAMT 2006 December 2006, pp. 57-58 (2006)

5. Dorn, J., Naz, T.: Structuring meta-search research by design patterns. In: ICSTC 2008 (March 2008)

6. Ferreira, J., Silva, A., Delgado, J.: A model-based approach to information retrieval systems development. In: Cheng, A. (ed.) Software Engineering and Application (November 2006)

7. Fons, J., Pelechano, V., Albert, M., Pastor, O.: Development of web applications from web enhanced conceptual schemas. In: Song, I.-Y., Liddle, S.W., Ling, T.W., Scheuermann, P. (eds.) ER 2003. LNCS, vol. 2813, pp. 232-245. Springer, Heidelberg (2003)

8. Ginige, A., Murugesan, S.: Guest editors' introduction: Web engineering - an introduction. IEEE MultiMedia 8(1), 14-18 (2001)

9. Jonkers, H., Steen, M.W.A., Heerink, L., Leeuwen, D.V., Telematica Instituut: Bridging BPM and MDE: On the Integration of BiZZdesigner and OptimalJ. In: Eclipse Summit Europe 2007 (October 2007)

10. Koch, N., Kraus, A., Cachero, C., Meliá, S.: Integration of business processes in web application models. Journal of Web Engineering 3(1), 22-49 (2004)

11. Liew, P., Kontogiannis, K., Tong, T.: A framework for business model driven development. In: STEP 2004, Washington, DC, USA, pp. 47-56. IEEE, Los Alamitos (2004)

12. Merialdo, P., Atzeni, P., Mecca, G.: Design and development of data-intensive web sites: The araneus approach. ACM Trans. Internet Techn. 3(1), 49-92 (2003)

13. Moulton, L.: Enterprise Search Markets and Applications. Capitalizing on Emerging Demand. Gilbane Group, Report \& Studies edition (June 2008)

14. Noll, J., Scacchi, W.: Specifying process-oriented hypertext for organizational computing. J. Netw. Comput. Appl. 24(1), 39-61 (2001)

15. Schmid, H.A., Rossi, G.: Modeling and designing processes in e-commerce applications. IEEE Internet Computing 8(1), 19-27 (2004)

16. Schwabe, D., Rossi, G., Barbosa, S.D.J.: Systematic hypermedia application design with OOHDM. In: Hypertext, pp. 116-128. ACM, New York (1996)

17. Torres, V., Pelechano, V.: Building business process driven web applications. In: Dustdar, S., Fiadeiro, J.L., Sheth, A.P. (eds.) BPM 2006. LNCS, vol. 4102, pp. 322-337. Springer, Heidelberg (2006)

18. Troyer, O.D., Casteleyn, S.: Modeling complex processes for web applications using wsdm. In: IWWOST 2003 (July 2003)

19. Web Models S.r.l. WebRatio (2008)

20. White, S.: Introduction to BPMN. OGM - BPM Initiative (2004)

21. Zhang, J., Qu, W., Du, L., Sun, Y.: A framework for domain-specific search engine: Design pattern perspective. In: IEEE Conf. on Systems, Man and Cybernetics, vol. 4(3881) (October 2003) 